\title{
Mesostructured ZnO/Au Nanoparticle Composites with Enhanced Photocatalytic Activity
}

Carina Bojer, ${ }^{1+}$ Judith Schöbel, ${ }^{2+}$ Thomas Martin, ${ }^{1}$ Thomas Lunkenbein, ${ }^{3}$ Daniel R. Wagner, ${ }^{1}$ Andreas Greiner, ${ }^{2,4}$ Josef Breu ${ }^{1,4^{*}}$ and Holger Schmalz ${ }^{2,4^{*}}$

${ }^{1}$ Lehrstuhl für Anorganische Chemie I, Universität Bayreuth, 95440 Bayreuth, Germany

E-mail: josef.breu@uni-bayreuth.de

${ }^{2}$ Lehrstuhl für Makromolekulare Chemie II, Universität Bayreuth, 95440 Bayreuth, Germany

E-Mail: holger.schmalz@uni-bayreuth.de

${ }^{3}$ Abteilung für Anorganische Chemie, Fritz-Haber Institut der Max-Planck Gesellschaft, 14195 Berlin, Germany

${ }^{4}$ Bavarian Polymer Institute (BPI), Universität Bayreuth, 95440 Bayreuth, Germany

${ }^{+}$C.B. and J.S. contributed equally to this work.

Dedicated to Axel H. E. Müller on the occasion of his $70^{\text {th }}$ birthday.

Abstract

Ease of catalyst separation from reaction mixtures represents a significant advantage in heterogeneous photocatalytic wastewater treatment. However, the activity of the catalyst strongly depends on its surface-to-volume ratio. Here, we present an approach based on cylindrical polybutadiene-block-poly(2-vinylpyridine) polymer brushes as template, which can be simultaneously loaded with zinc oxide $(\mathrm{ZnO})$ and gold $(\mathrm{Au})$ nanoparticles. Pyrolytic template removal of the polymer yields in mesostructured $\mathrm{ZnO} / \mathrm{Au}$ composites, showing higher efficiencies in the photocatalytic degradation of ciprofloxacin and levofloxacin (generic antibiotics present in clinical wastewater) as compared to neat mesostructured $\mathrm{ZnO}$. Upscaling of the presented catalyst is straightforward promising high technical relevance.

Keywords

Mesostructure, cylindrical polymer brushes, wastewater treatment, photocatalyst, nanowires

\section{Introduction}


The removal of pharmaceuticals from clinical wastewater is a burning issue in environmental science. Efficient removal of active drugs like, antibiotics, cytostatics, beta-blockers or antiphlogistics, is required before treatment by standard biological purification techniques in sewage plants.[1-5] For instance, ciprofloxacin, a widely employed antibiotic from the fluoroquinolone group, is problematic due to its gentoxicity.[6,7] Consequently, high stability, low biodegradability and toxicity of these active agents limit the use of mild biological degradation processes and there is a demand for chemical oxidation.[8] Advanced oxidation processes (AOP), which are based on the formation of highly reactive hydroxyl radicals, provide promising results in the catalytic degradation of organic pollutants. In particular, photocatalytic AOPs are of special interest, as in addition to UV light only a transition metal oxide catalyst, e.g. titanium dioxide $\left(\mathrm{TiO}_{2}\right)$ or zinc oxide $(\mathrm{ZnO})$, is needed.[9-11] If solar energy, consisting of UV and visible light, is applied to the catalyst, an enhancement of the activity by doping the transition metal oxide with noble metal nanoparticles (NPs), e.g., gold, platinum or silver, has been observed. This effect is attributed to the localized surface plasmon resonance of the noble metal NPs, which causes an increased absorption of visible light and a charge-transfer to the transition metal oxide.[12-15]

With respect to clinical wastewater (typical $\mathrm{pH}=8$ ), the catalyst has to fulfil different requirements.[16,17] Most pharmaceuticals are rendered soluble in body fluids by deprotonated carboxylic acid groups. For ciprofloxacin the isoelectric point of the zwitterion is at a $\mathrm{pH}$ value of 7.4.[18] Thus, a catalyst bearing a positive surface charge at a $\mathrm{pH}$ of 8 is advantageous, as this will enhance the electrostatic interactions between antibiotic and catalyst. Previous studies showed that in contrast to Degussa $\mathrm{P} 25, \mathrm{a} \mathrm{TiO}_{2}$ catalyst with its point of zero charge (PZC) at a $\mathrm{pH}$ of $6.9, \mathrm{ZnO}$ fulfils this requirement as its $\mathrm{PZC}$ is 9.2. $[19,20]$ Another important criterion for increasing catalytic activity is the accessible surface area of the catalyst. Using nanomaterials, like nanoparticles, -tubes, or -wires, the active surface can be maximized due to their high surface-to-volume ratio, resulting in an increased performance for example in environmental remediation.[4] However, the reusability of NP catalysts is limited due to the inherently difficult separation of NPs from a fluid reaction media. Applying polymer templates for the controlled assembly of NPs into mesostructured hybrid materials represents a good compromise between preserving a high accessible surface area while allowing for filtration of the catalyst (Scheme 1). In particular, cylindrical polymer brushes (CPBs) proved to be promising template materials, as their composition and functionality can be tailored to meet the demands for an efficient mesostructuring of NPs.[21-24] As shown with different transition metal oxides, like $\mathrm{TiO}_{2}$ or $\mathrm{WO}_{3}$, these supports are able to prevent segregation and, therefore, a loss of surface area.[25-27] Pyrolytic removal of the polymeric template leads to mesostructured one-dimensional aggregates where adjacent NPs become connected via necks during sintering. $[28,29]$ The resulting nonwoven structures provide sufficient mechanical stability to be used in continuous flow cells, while at the same time offering a high accessible surface area.[30]

Herein, we report the synthesis of mesostructured $\mathrm{ZnO} / \mathrm{Au}$ NP composites for the efficient detoxification of hospital wastewater, exemplified on the photocatalytic degradation of ciprofloxacin and levofloxacin. To this end, cationic CPBs, consisting of a cross-linked polybutadiene (PB) core and a quaternized poly(2-vinylpyridine) (P2VP) corona, were used as template for the simultaneous mesostructuring of $\mathrm{ZnO}$ and Au NPs. Pyrolytic removal of the polymeric template leads to hierarchically structured nonwovens composed of catalytically active $\mathrm{ZnO} / \mathrm{Au}$ nanowires (Scheme 1). 


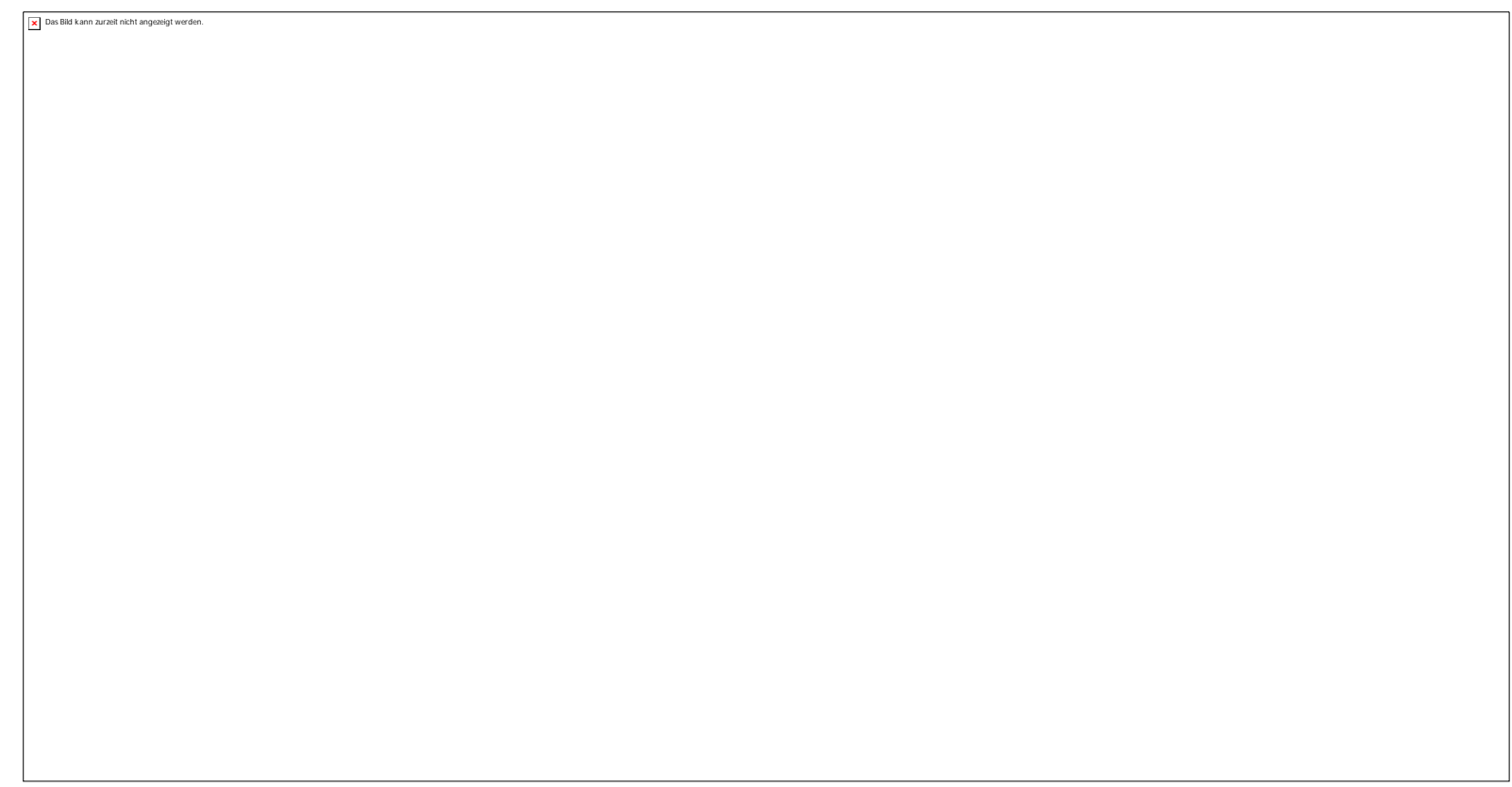

Scheme 1. Self-assembly of a BV diblock copolymer (A) results in a cylindrical bulk morphology with hexagonally packed PB cylinders in a P2VP matrix (B). Crosslinking the PB cylinders and quaternization of the P2VP corona blocks produces cationic CPBs (C). These CPBs are used as templates for the simultaneous loading with $\mathrm{ZnO}$ and Au NPs (D). The resulting hybrid (E) is transformed into a mesostructured nonwoven $(F)$ consisting of $\mathrm{ZnO} / \mathrm{Au}$ nanowires by calcination. 


\section{Experimental Part}

\subsection{Chemicals}

All chemicals were purchased from Sigma-Aldrich (Germany) and used as received if not otherwise noted.

\subsection{Synthesis of the BV diblock copolymer}

The polybutadiene-block-poly(2-vinylpyridine) (BV) diblock copolymer was synthesized by sequential living anionic polymerization in THF with sec-butyllithium as initiator, as published elsewhere.[28] The molecular weight and the composition of the BV diblock copolymer were determined by a combination of ${ }^{1} \mathrm{H}-\mathrm{NMR}$ spectroscopy (Bruker Ultrashield AC300) and MALDI-TOF MS (Bruker Reflex III) and resulted in $\mathrm{B}_{19} \mathrm{~V}_{81}{ }^{60}$ (subscripts denote the mass fraction of the corresponding block in wt-\% and the superscript gives the overall molecular weight in $\mathrm{kg} \mathrm{mol}^{-1}$ ). The molar mass dispersity of the BV diblock copolymer was determined by THF-SEC to $\bigoplus_{M}=1.02$.

\subsection{Formation of the cationic CPB template}

Typically, $1 \mathrm{~g}$ of a $\mathrm{B}_{19} \mathrm{~V}_{81}{ }^{60}$ film with $0.1 \mathrm{~g}$ of the photoinitiator Lucirin TPO (BASF) was prepared by solvent-casting from chloroform. The resulting hexagonally ordered film was crosslinked using a UV hand lamp (Hoenle, $2 \mathrm{~h}$ per side). The crosslinked $\mathrm{B}_{19} \mathrm{~V}_{81}{ }^{60}$ film was dissolved in $200 \mathrm{~mL}$ chloroform and $3 \mathrm{~mL}$ of an iodomethane solution (99\%) was added dropwise for quaternization of the poly(2-vinylpyridine) (P2VP) block. The solvent and the iodomethane solution were allowed to evaporate. The quaternized product $\left(\mathrm{BV}_{\mathrm{q}}\right)$ was washed with pentane, dissolved in water and freeze-dried.

\subsection{Synthesis of the hybrid material and calcination}

As an example, for the synthesis of the $\mathrm{ZnO} / \mathrm{Au}_{1} 12$ hybrid material $200 \mathrm{mg}$ of $\mathrm{BV}_{\mathrm{q}}$ was dissolved in $200 \mathrm{~mL}$ DMSO. To this solution, $6 \mathrm{~mL}$ of tetrachloroauric acid trihydrate (Alfa Aesar, $0.1 \mathrm{M}$ in THF) was added. Subsequently, $3 \mathrm{~mL}$ of a sodium borohydride solution (1 M in deionized water) was added and the colour changed to deep purple. Immediately, $125 \mathrm{~mL}$ of an acetate-stabilized ZnO NP dispersion $\left(6.9 \mathrm{~g} \mathrm{~L}^{-1}\right.$ in ethanol, synthesis reported in Ref. 30) was added. The reaction mixture was stirred for $10 \mathrm{~min}$. The purple precipitate was isolated by centrifugation ( $3 \mathrm{~min}, 7000 \mathrm{rpm}$ ) and washed 5 times with ethanol and one time with deionized water and then freeze-dried. The details of all synthesized materials are summarized in Table S1. Heat treatment at $490^{\circ} \mathrm{C}$ for $5 \mathrm{~min}$ in a tube furnace (heating/cooling rate $180 \mathrm{~K} \mathrm{~h}^{-1}$ ) under air lead to the removal of the organic CPB template and sintering into an all-inorganic nonwoven composite structure. 


\subsection{Photocatalytic degradation}

Photocatalytic degradation of anionic antibiotics (ciprofloxacin and levofloxacin) was realized in a self-constructed continuous flow reactor (Scheme S1). The synthesized $\mathrm{ZnO} / \mathrm{Au}$ nanowire photocatalyst $(15 \mathrm{mg}$ ) was drop coated on a glass microfiber filter (Whatman, $37 \mathrm{~mm}$ diameter) and $400 \mathrm{~mL}$ of the ciprofloxacin or levofloxacin solution $\left(c=2 \cdot 10^{-5} \mathrm{~mol} \mathrm{~L}^{-1}\right.$, $\mathrm{pH}=8$ (borate/HCl buffer, Titrisol)) was circulated at a flow rate of $12 \mathrm{~L} \mathrm{~h}^{-1}$. The degradation with solar spectrum ( $300 \mathrm{~W}$ Xenon lamp, AM1.5 filter, $1000 \mathrm{~W} \mathrm{~m}^{-2}$ ) was monitored using UVVis spectroscopy (samples taken every 5-15 min). The experiments were done without catalyst as a reference, with Degussa P25, ZnO nanotubes and ZnO/Au nanowire composites with different $\mathrm{ZnO} / \mathrm{Au}$ ratios. All catalysts were tested at least three times to guarantee reproducible results.

\subsection{Instruments}

Powder X-ray diffraction (PXRD) measurements were done using a STOE Stadi P diffractometer. CuK ${ }_{\alpha 1}$ radiation and a Mythen $1 \mathrm{~K}$ silicon strip-detector were used. Rietveld analysis was done with TOPAS Academic (fundamental parameters approach for profile fitting). The crystallite sizes were determined using implemented macros (elliptical particles) and the composition was estimated using quantitative Rietveld-refinement.

Transmission electron microscopy (TEM) micrographs were acquired using a Zeiss 922 Omega EFTEM (Zeiss NTS GmbH, Oberkochen, Germany), operating at an accelerating voltage of $200 \mathrm{kV}$. Zero-loss filtered images were taken with a bottom-mounted CCD camera system (Ultrascan 1000, Gatan). The micrographs were processed by the digital imaging processing software Gatan Digital Micrograph 3.9 for GMS 1.4. The samples were prepared by drop-coating of dilute dispersions of the hybrid materials/composites in ethanol ( $c=$ $0.1 \mathrm{~g} \mathrm{~L}^{-1}$ ) onto carbon-coated copper grids, followed by blotting with a filter paper.

High resolution TEM and local energy dispersive X-ray (EDX) maps were recorded on a double corrected JEOL ARM-200CF equipped with a high angle Silicon Drift EDX detector with a solid angle of up to 0.98 sterradians.

Scanning electron microscopy (SEM) images and energy dispersive X-ray analysis (EDX) data were obtained with a LEO 1530 FESEM scanning electron microscope equipped with a field emission cathode.

A Quantachrome Autosorb 1 was used to determine the surface area with $\mathrm{N}_{2}$ at $77 \mathrm{~K}$ after degassing the sample at $363 \mathrm{~K}$ for $12 \mathrm{~h}$.

UV-Vis absorption spectra were measured with a Varian Cary 300 spectrometer using Hellma precision cells made of Quartz SUPRASIL (type 100-QS, light path $10 \mathrm{~mm}$ ).

Thermal gravimetric analysis (TGA) was performed on a Netzsch TG 209F1 Libra under synthetic air. The samples were heated from $25-800{ }^{\circ} \mathrm{C}$ at a heating rate of $10 \mathrm{~K} \mathrm{~min}^{-1}$.

The PZC was determined using a ParticleMetrix StabiSizer PMX 200C. 50 mg catalyst in $10 \mathrm{~mL}$ water with a $\mathrm{pH}$ of 10.5 (adjusted with $0.01 \mathrm{M} \mathrm{NaOH}$ ) were titrated with $0.05 \mathrm{M} \mathrm{HCl}$ until the streaming potential was zero.

\section{Results and Discussion}


$\mathrm{ZnO} / \mathrm{Au}$ hybrids were prepared by employing cationic CPBs as template for the simultaneous loading with $\mathrm{Au}$ and $\mathrm{ZnO}$ NPs (Scheme 1). The cationic CPBs were obtained from a polybutadiene-block-poly(2-vinylpyridine) (BV) diblock copolymer $\left(\mathrm{B}_{19} \mathrm{~V}_{81}{ }^{60}\right.$ : subscripts denote the mass fraction of the corresponding block in wt-\% and the superscript gives the molecular weight in $\mathrm{kg} \mathrm{mol}^{-1}$ ), which forms a bulk morphology with hexagonally arranged PB cylinders embedded in a P2VP matrix (Scheme 1A, B). Subsequent photo-crosslinking of the PB cylinders followed by dissolution and quaternization of the P2VP block resulted in the desired CPBs with a positively charged corona (Scheme 1C). The Au NPs were generated in-situ by reduction of tetrachloroauric acid trihydrate and as a $\mathrm{ZnO}$ source phase pure, acetatestabilized ZnO NPs with a number-weighted hydrodynamic diameter of $D_{h}=3.2 \mathrm{~nm}$ were used (Scheme 1D, E; Table S1). Details on the synthesis and characterization of the used monodisperse ZnO NPs can be found elsewhere.[30] To investigate the effect of the Au content on the photocatalytic degradation of antibiotics (ciprofloxacin and levofloxacin), four different $\mathrm{ZnO} / \mathrm{Au}$ hybrid materials (8, 12, 15 and $26 \mathrm{wt}$-\% Au with respect to the overall amount of inorganic material in the hybrid) were synthesized. The notation $\mathrm{ZnO} / \mathrm{Au}$ _x , with $\mathrm{x}$ denoting the Au content in wt-\%, will be used in the following. The total amount of $\mathrm{ZnO}$ and $\mathrm{Au}$ immobilized by the cationic CPB template was determined by TGA measurements to 7479 wt-\% (Fig. S1). This is in good agreement with the employed amounts of the CPB template, ZnO NP and Au precursor in the synthesis of the hybrid materials (80 wt-\% CPB template).

The TEM micrographs of the obtained hybrids reveal the controlled co-assembly of $\mathrm{ZnO}$ and $\mathrm{Au}$ NPs under preservation of the cylindrical structure of the cationic CPB template (Fig. 1). The cylindrical PB core of the CPB template can be clearly identified in the corresponding greyscale analysis for the $\mathrm{ZnO} / \mathrm{Au} \_12$ hybrid, shown in the inset of Fig. 1B. 


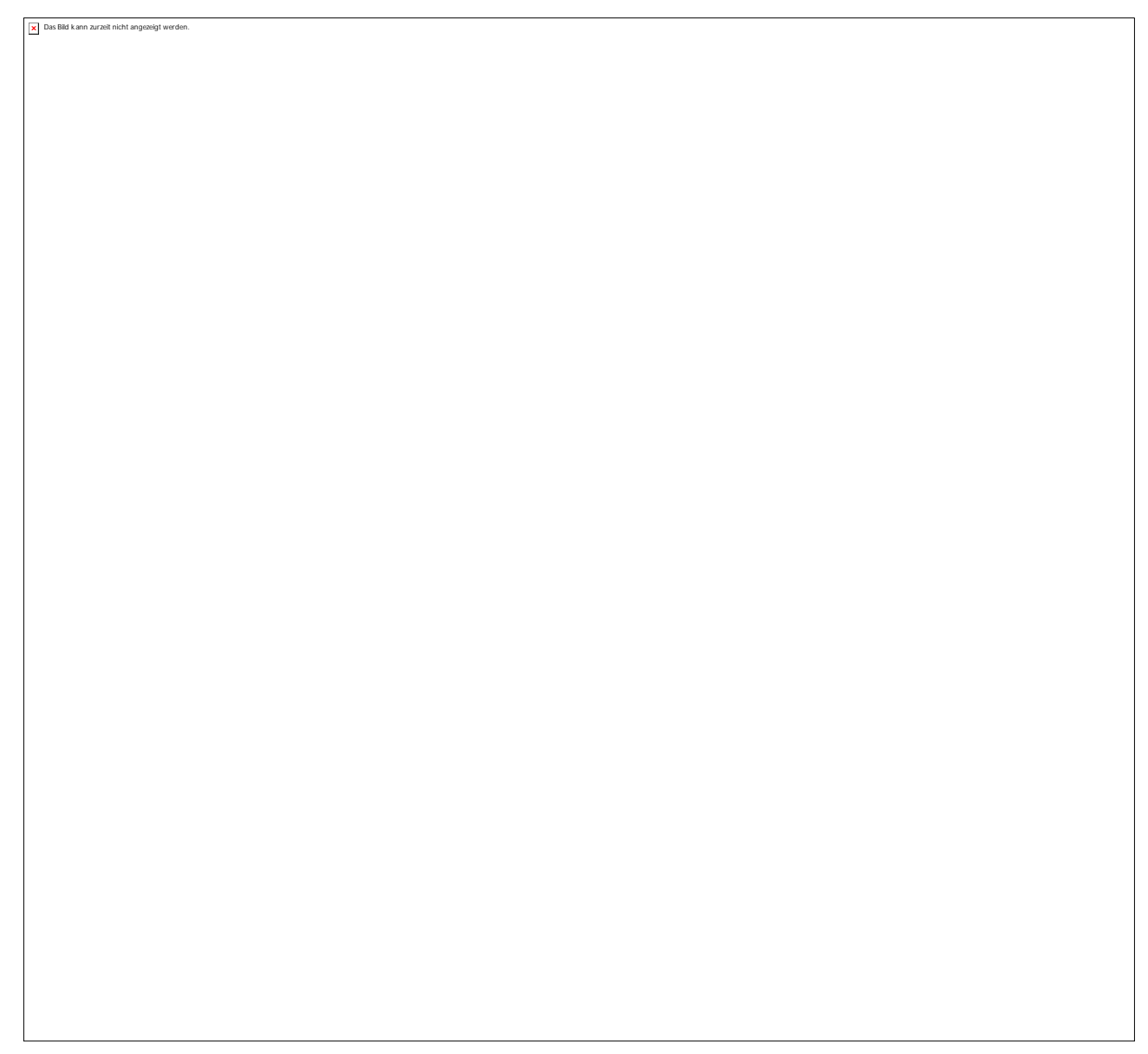

Fig. 1. TEM images of the $\mathrm{ZnO} / \mathrm{Au} \_8(\mathrm{~A}), \mathrm{ZnO} / \mathrm{Au} \_12$ (B; inset in $\mathrm{B}$ : grey scale analysis), ZnO/Au_15 (C) and the $\mathrm{ZnO} / \mathrm{Au} \_26$ hybrid materials (D).

\subsection{Formation of $\mathrm{ZnO} / \mathrm{Au}$ composite nanowires}

$\mathrm{ZnO} / \mathrm{Au}$ composite nanowires were obtained by pyrolysis of the $\mathrm{ZnO} / \mathrm{Au}$ hybrid cylinders in air at $490^{\circ} \mathrm{C}$. After template removal, the one-dimensional structure of the $\mathrm{CPB}$ template was retained, resulting in the formation of mesostructured $\mathrm{ZnO} / \mathrm{Au}$ composite nonwovens (Fig. 2A-D). Only for the ZnO/Au_26 nonwoven a partial agglomeration of the hybrid cylinders upon calcination can be observed. The composition of the obtained $\mathrm{ZnO} / \mathrm{Au}$ nanowires was determined by EDX measurements (Table S2) and is in good agreement with the used amount of educts. Elemental analysis (Table S3) and TGA measurements (Fig. S1) confirm the absence of organic material after pyrolysis (less than $0.2 \%$ ), indicating the complete removal of the organic CPB template. The mesostructured nonwovens show high specific surface areas of $25-48 \mathrm{~m}^{2} \mathrm{~g}^{-1}$, as determined by nitrogen physisorption (Fig. S2, Table S4). The high-angle annular dark-field scanning transmission electron microscopy (HAADF-STEM) image of ZnO/Au_12 nanowires (Fig. 2E) confirms the retention of the wirelike mesostructure. In HAADF-STEM the contrast is due to Rutherford scattering, which is almost proportional to $Z^{2}$. Thus, $\mathrm{Au}$ (bright) and $\mathrm{ZnO}$ (grey) moieties are directly 
distinguishable and can further be identified by an energy dispersive X-ray (EDX) map of the same particles (Fig. 2E, inset). The HAADF-STEM image reveals a partial wetting of the Au NPs on top of the $\mathrm{ZnO}$ nanocrystals, thus, increasing the $\mathrm{Au} / \mathrm{ZnO}$ perimeter. In addition, the HR-TEM image in Fig. 3F highlights a representative mesostructure of the nanowires, which is composed of randomly oriented, individual $\mathrm{ZnO}$ nanocrystals that form intraparticular mesopores. Those intraparticular mesopores may ensure a higher mass transport in the photocatalytic liquid phase detoxification reaction. Moreover, the $\mathrm{ZnO}$ nanowires are partially decorated by isolated Au nanoparticles.

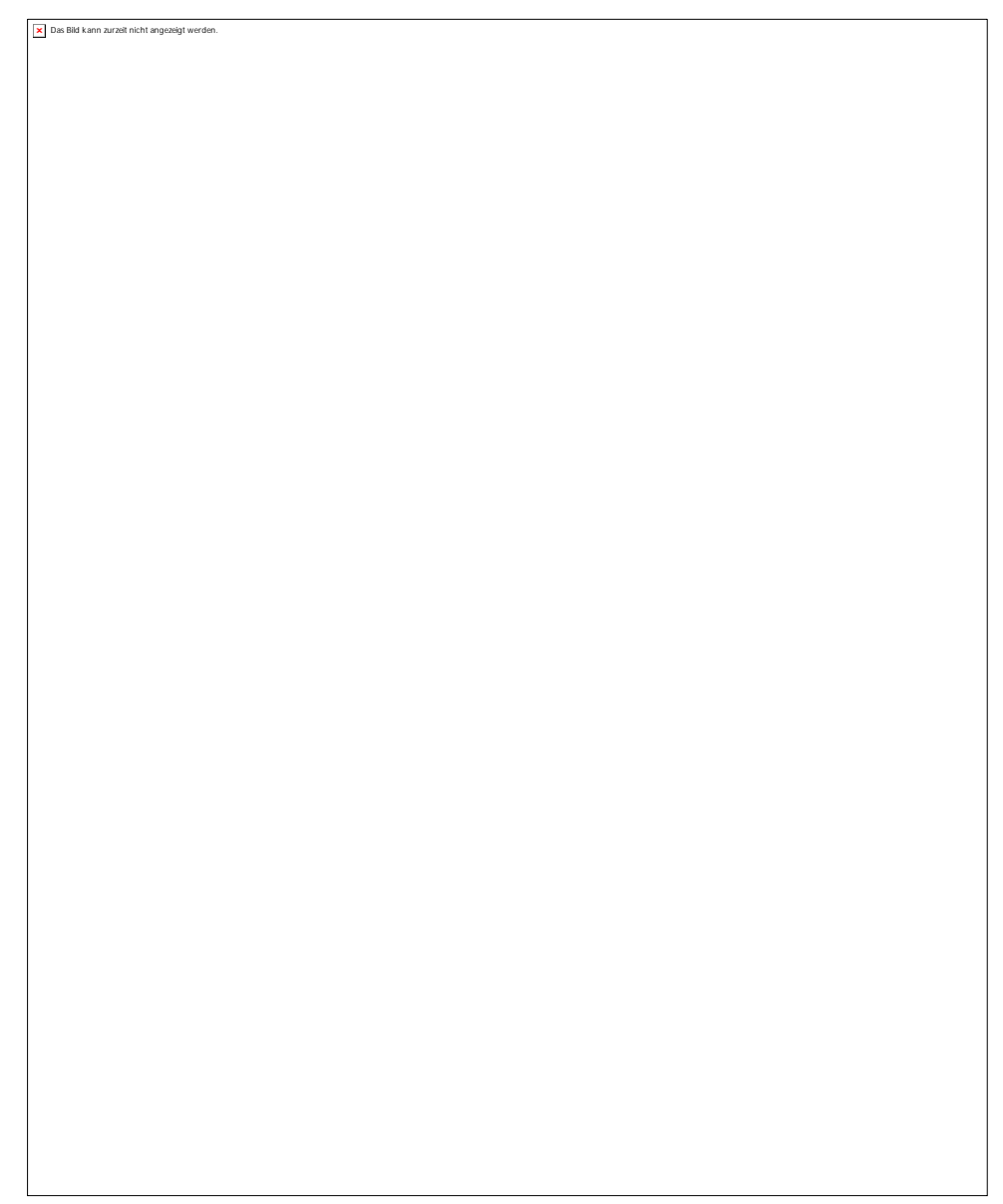

Fig. 2. SEM images of $\mathrm{ZnO} / \mathrm{Au}$ _8 (A), ZnO/Au_12 (B), ZnO/Au_15 (C) and $\mathrm{ZnO} / A u \_26$ nanowires (D). HAADF-STEM image of ZnO/Au_12 nanowires (E, inset: EDX map of the same particle; white - ZnO; red - Au). HR-TEM image of $\mathrm{ZnO} / \mathrm{Au}_{-} 12$ nanowires $(\mathrm{F})$; the inset denotes the Fast Fourier Transform (FFT) of the region that is labelled with $\mathrm{Au}$.

In order to get a closer insight into the calcination process and its impact on the $\mathrm{Au}$ and $\mathrm{ZnO}$ $\mathrm{NP}$ sizes, the morphological changes for the $\mathrm{ZnO} / \mathrm{Au}$ _12 and $\mathrm{ZnO} / \mathrm{Au}$ _26 hybrid cylinders upon calcination were studied by PXRD (Fig. 3A, B). The PXRD data show a halo in the range of $2 \theta=20-30^{\circ}$, which is characteristic for the amorphous CPB template. The crystalline reflections of the hybrid materials can be assigned to $\mathrm{ZnO}$ and $\mathrm{Au}$, respectively. For ZnO/Au_12 Rietveld refinement yields average coherent scattering domains of 
3.4(2) x 3.7(2) nm for $\mathrm{ZnO}$ and 3.5(2) x 4.9(2) nm for Au, respectively (Fig. S3, Table S5). For $\mathrm{ZnO} / \mathrm{Au} \_26$ domain sizes of 3.5(2) $\times 4.4(3) \mathrm{nm}$ for $\mathrm{ZnO}$ and 3.7(6) $\times 5.3(4) \mathrm{nm}$ for $\mathrm{Au}$ (Fig. S4 and Table S6) were obtained.
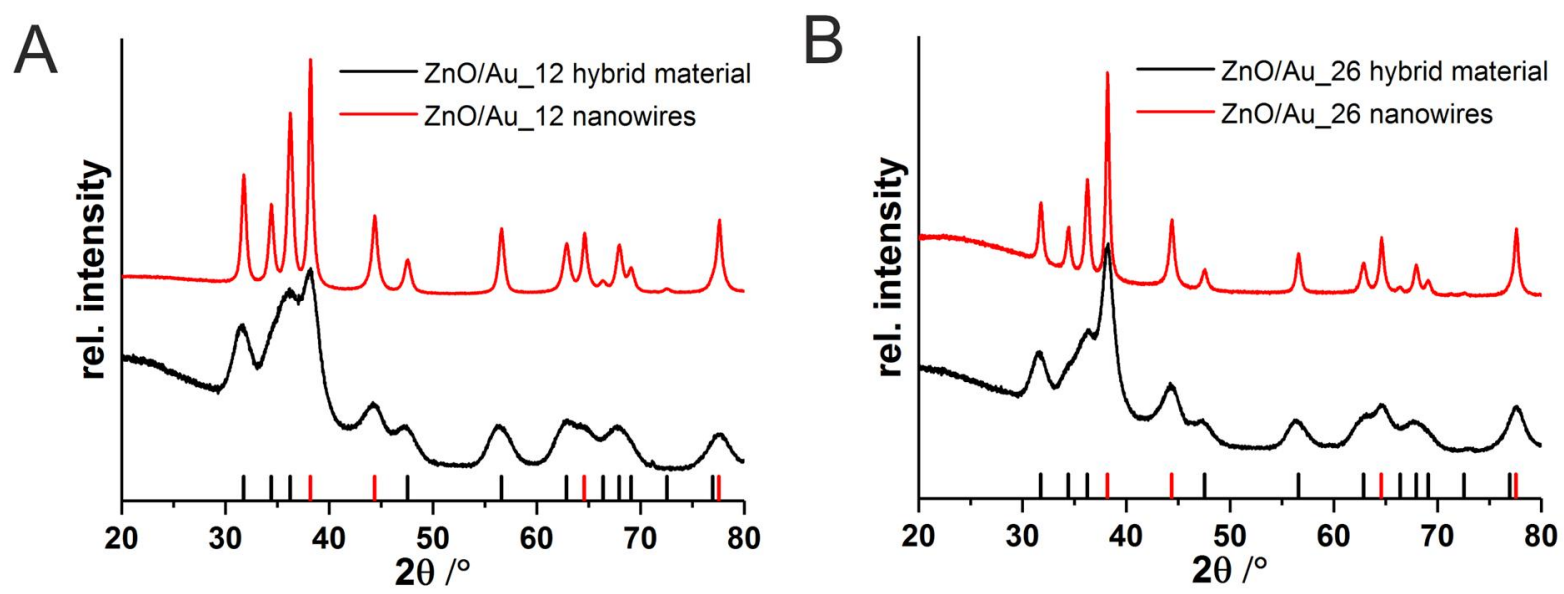

Fig. 3. PXRD patterns of $\mathrm{ZnO} / \mathrm{Au} \_12(\mathrm{~A})$ and $\mathrm{ZnO} / \mathrm{Au} \_26$ (B) hybrid cylinders and nanowires with the reference cards of $\mathrm{ZnO}$ (black ticks PDF-Nr.: 00-036-145) and Au (red ticks PDF-Nr.: 00-004-0784).

The decrease of the full width at half maxima of PXRD peaks ( $\mathrm{ZnO} / \mathrm{Au}$ _12, Fig. 3A; $\mathrm{ZnO} / \mathrm{Au} \_26$, Fig. 3B) indicates Ostwald ripening upon pyrolysis. For $\mathrm{ZnO} / \mathrm{Au} \_12$ Rietveld analysis confirms the growth to a crystallite size of $12.5(4) \times 15.5(4) \mathrm{nm}$ for $\mathrm{ZnO}$ and $17.5(4) \times 24.4(5) \mathrm{nm}$ for Au, respectively (Fig. S5 and Table S7). A similar trend is observed for ZnO/Au_26, showing increased crystallite sizes of 14.5(8) $\times 16.9(8)$ for $\mathrm{ZnO}$ and $16.9(3) \times 23.7(5)$ for Au (Fig. S6 and Table S8). The composition of the calcinated ZnO/Au_12 and ZnO/Au_26 composites is confirmed by Rietveld analysis and is in good agreement with the educt stoichiometry (12 wt-\% Au and $26 \mathrm{wt} \% \mathrm{Au}$, respectively) and EDX measurements (Table S2). 


\subsection{Photocatalytic degradation of negatively charged antibiotics}

The photocatalytic degradation of ciprofloxacin and levofloxacin (isoelectric points (zwitterion): 7.4 and 6.8, respectively)[18], applying the $\mathrm{ZnO} / \mathrm{Au}$ composite nanowires as catalysts, was studied using a continuous flow reactor. For comparative studies, neat $\mathrm{ZnO}$ nanotubes and Degussa P25 ( $\mathrm{TiO}_{2}$ based photocatalyst) were used. In the continuous flow reactor, $3.5 \mathrm{ppm}$ of the catalyst material (with respect to ciprofloxacin or levofloxacin) was placed onto a filter paper to guarantee a homogeneous flow and the photocatalytic degradation of the two antibiotics was monitored at $\mathrm{pH}=8$ for 120 min applying terrestrial solar spectrum. No significant degradation was observed in the absence of a catalyst (Fig. S7A). The $\mathrm{ZnO} / \mathrm{Au}$ _12 composite nanowires revealed the highest efficiency in the photocatalytic degradation of ciprofloxacin with a degradation of $16 \%$ in 120 min (Fig. 4A). For composite nanowires with a lower ( 8 wt-\%) as well as higher (15 and 26 wt-\%) Au content a significantly lower conversion was observed. Similar results were obtained for the degradation of levofloxacin (Fig. S8A), i.e., the $\mathrm{ZnO} / \mathrm{Au}_{1} 12$ composite nanowires showed the highest efficiency in photocatalytic degradation within the tested catalysts. The inferior behaviour for higher Au-loading can be attributed to electron-hole recombination centres, which limit the synergistic effect of the $\mathrm{ZnO} / \mathrm{Au}$ composite.[31] The reduced performance of $\mathrm{ZnO} / \mathrm{Au}_{1} 15$ and $\mathrm{ZnO} / \mathrm{Au} \_26$ also indicates that thermal effects due to the strong localized surface plasmon resonance absorption of Au NPs can be neglected, as in this case a higher activity would be expected for composites with higher Au contents. In comparison to previous studies on the degradation of ciprofloxacin with neat $\mathrm{ZnO}$ nanotubes,[30] the $\mathrm{ZnO} / \mathrm{Au}$ _12 composite material shows a ca. $40 \%$ improved performance, as with the $\mathrm{ZnO}$ nanotubes a conversion of only $12 \%$ could be achieved under identical reaction conditions (Fig. S7A). A similar effect was observed for the photocatalytic degradation of levofloxacin (Fig. S8A).

The photocatalytic activity of the $\mathrm{ZnO} / \mathrm{Au}_{-} 12$ nanowires in the degradation of ciprofloxacin is moreover significantly improved with respect to Degussa P25, a commercial $\mathrm{TiO}_{2}$ based photocatalyst (Fig. S7A). The advantage of ZnO/Au nanowire composites (PZC: 8.8) over Degussa P25 (PZC: 6.9)[19, 20] can be attributed to the opposite surface charge of ZnO based catalysts and ciprofloxacin (isoelectric point (zwitterion): 7.4 )[18] at $\mathrm{pH}=8$ that favours drug adsorption on the catalyst surface. Any influence attributed to differences in the specific surface area of the catalysts can be ruled out, as the $\mathrm{ZnO} / \mathrm{Au}_{1} 12$ catalyst, showing the highest activity, exhibits the lowest specific surface area $\left(25 \mathrm{~m}^{2} \mathrm{~g}^{-1}\right.$, Table S4). 

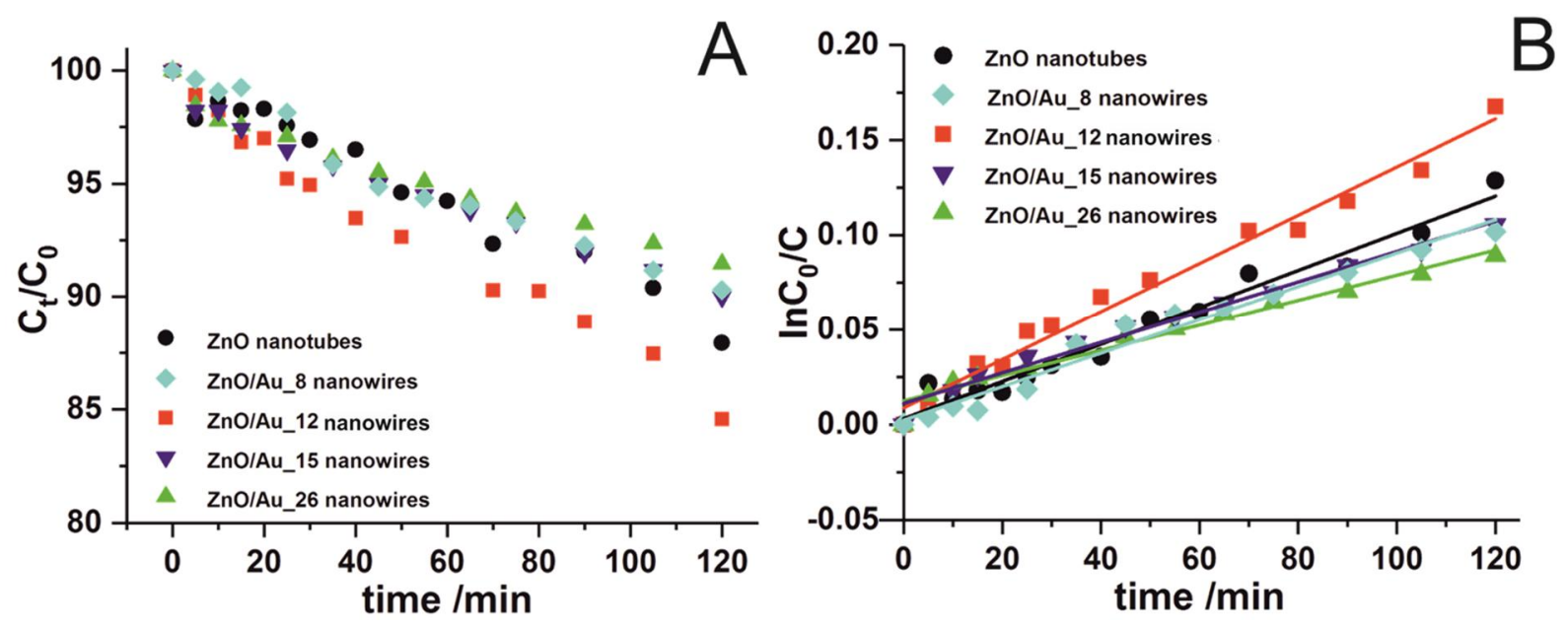

Fig. 4. Time-dependent decrease of ciprofloxacin concentration during irradiation with terrestrial solar spectrum (A) and corresponding first-order kinetics plots (B) for $\mathrm{ZnO}$ nanotubes and $\mathrm{ZnO} / \mathrm{Au}$ composite nanowires.

As the photocatalytic degradation of ciprofloxacin using a $\mathrm{ZnO}$ or $\mathrm{TiO}_{2}$ based catalyst already showed, this reaction follows a pseudo first-order kinetics and the apparent rate constants $k$ can be calculated from the slope of the corresponding first-order kinetics plot (Fig. 4B). The $k^{\prime}$ value for $\mathrm{ZnO} / \mathrm{Au}$ _12 nanowires is $1.3 \cdot 10^{-3} \mathrm{~min}^{-1}$. Compared with the $k^{\prime}$ value of neat $\mathrm{ZnO}$ nanotubes $\left(k^{*}=9.6 \cdot 10^{-4} \mathrm{~min}^{-1}\right)$ the $\mathrm{ZnO} / \mathrm{Au}_{-} 12$ catalyst is 1.4 times faster. With reference to previous results, the ciprofloxacin degradation with $\mathrm{ZnO} / \mathrm{Au}_{-} 12$ nanowires is 3.7 times faster as compared to the standard Degussa P25 (Fig. S7B, Table S9). In case of levofloxacin, a 1.6 times faster degradation was observed for $\mathrm{ZnO} / \mathrm{Au}_{-} 12$ nanowires (Fig. S8B, Table S10). Conseqeuently, combining the electrostatically favoured adsorption of the antibiotics on the positively charged $\mathrm{ZnO}$ surface with the enhancement of Au NPs in mesostructured $\mathrm{ZnO} / \mathrm{Au} \_12$ nonwovens has a distinct synergistic effect on the photocatalytic degradation. This results in an increase in degradation rate of about $40 \%$ for ciprofloxacin and $60 \%$ for levofloxacin, respectively, compared to neat $\mathrm{ZnO}$ nanotubes.

\section{Conclusion}

In this study, we used cationic CPBs as template for mesostructuring two different types of NPs with the aim of taking advantage of synergistic effects for the photocatalytic degradation of antibiotics in wastewater. $\mathrm{ZnO}$ and Au NPs were assembled simultaneously in a random fashion on cationic CPBs, resulting in mesostructured, nonwoven-like networks of $\mathrm{ZnO} / \mathrm{Au}$ nanowires after pyrolytic template removal. The $\mathrm{ZnO} / \mathrm{Au}$ nanowires with 12 wt-\% Au show a significantly enhanced efficiency in the photocatalytic degradation of ciprofloxacin and levofloxacin with terrestrial solar spectrum, both with respect to neat $\mathrm{ZnO}$ nanotubes and 
Degussa P25 NPs, respectively. This can be ascribed to the improved adsorption of the antibiotics, which are negatively charged at the slightly basic $\mathrm{pH}$ of hospital wastewater, on the positively charged $\mathrm{ZnO}$ surface combined with the enhancement due to the localized surface plasmon resonance of the Au NPs. The assembly of different types of NPs allows not only the synthesis of tailor-made catalyst systems for photocatalytical processes, but can also be transferred to other NP combinations with interesting catalytic properties. The nonwoven structure of the catalyst ensures an easy recovery from the fluid reaction medium and, thus, excellent reusability, making this system highly interesting for technical applications.

\section{Acknowledgement}

This work was funded by the Collaborative Research Centre (SFB) 840. The authors thank Professor R. Schlögl (Fritz-Haber-Institute, Berlin) for access to HR-TEM. We appreciate the support of the Keylab for Optical and Electron Microscopy of the Bavarian Polymer Institute (BPI). J.S. acknowledges support by the Graduate School of the University of Bayreuth.

\section{References}

[1] N.M. Vieno, H. Härkki, T. Tuhkanen, L. Kronberg, Occurrence of Pharmaceuticals in River Water and Their Elimination in a Pilot-Scale Drinking Water Treatment Plant, Environ. Sci. Technol. 41 (2007) 5077-5084.

[2] E. Carraro, S. Bonetta, C. Bertino, E. Lorenzi, S. Bonetta, G. Gilli, Hospital Effluents Management: Chemical, Physical, Microbiological Risks and Legislation in Different Countries, J. Environ. Manage. 168 (2016) 185-199.

[3] J. Rivera-Utrilla, M. Sánchez-Polo, M.Á. Ferro-García, G. Prados-Joya, R. OcampoPérez, Pharmaceuticals as Emerging Contaminants and their Removal from Water. A Review, Chemosphere 93 (2013) 1268-1287.

[4] M.M. Khin, A.S. Nair, V.J. Babu, R. Murugan, S. Ramakrishna, F. Yinjun, L. Junkang, C.W. Zhou, J. Choi, J. Paek, K. Lee, Y.S. Lee, D.H. Jeong, M.H. Cho, A Review on Nanomaterials for Environmental Remediation, Energy Environ. Sci. 5 (2012) 8075819.

[5] A. Alsbaiee, B.J. Smith, L. Xiao, Y. Ling, D.E. Helbling, W.R. Dichtel, Rapid Removal of Organic Micropollutants from Water by a Porous $\beta$-Cyclodextrin Polymer, Nature 529 (2016) 190-194.

[6] D.G.J. Larsson, C. de Pedro, N. Paxeus, Effluent from Drug Manufactures Contains Extremely High Levels of Pharmaceuticals, J. Hazard. Mater. 148 (2007) 751-755.

[7] J.W. Beaber, B. Hochhut, M.K. Waldor, SOS Response Promotes Horizontal Dissemination of Antibiotic Resistance Genes, Nature 427 (2004) 72-74.

[8] A. Cincinelli, T. Martellini, E. Coppini, D. Fibbi, A. Katsoyiannis, Nanotechnologies for Removal of Pharmaceuticals and Personal Care Products from Water and Wastewater. A Review, J. Nanosci. Nanotechnol. 15 (2015) 3333-3347.

[9] U.I. Gaya, A.H. Abdullah, Heterogeneous Photocatalytic Degradation of Organic Contaminants over Titanium Dioxide: A Review of Fundamentals, Progress and 
Problems, J. Photochem. Photobiol. C 9 (2008) 1-12.

[10] N.S. Lewis, Toward Cost-Effective Solar Energy Use, Science 315 (2007) 798-801.

[11] K.M. Lee, C.W. Lai, K.S. Ngai, J.C. Juan, Recent Developments of Zinc Oxide Based Photocatalyst in Water Treatment Technology: A Review, Water Res. 88 (2016) 428448.

[12] R. Georgekutty, M.K. Seery, S.C. Pillai, A Highly Efficient Ag-ZnO Photocatalyst: Synthesis, Properties, and Mechanism, J. Phys. Chem. C 112 (2008) 13563-13570.

[13] A. Primo, A. Corma, H. García, Titania Supported Gold Nanoparticles as Photocatalyst, Phys. Chem. Chem. Phys. 13 (2011) 886-910.

[14] D. Tsukamoto, Y. Shiraishi, Y. Sugano, S. Ichikawa, S. Tanaka, T. Hirai, Gold Nanoparticles Located at the Interface of Anatase/Rutile $\mathrm{TiO}_{2}$ Particles as Active Plasmonic Photocatalysts for Aerobic Oxidation, J. Am. Chem. Soc. 134 (2012) 63096315.

[15] R.M. Mohamed, D. McKinney, M.W. Kadi, I.A. Mkhalid, W. Sigmund, Platinum/Zinc Oxide Nanoparticles: Enhanced Photocatalysts Degrade Malachite Green Dye Under Visible Light Conditions, Ceram. Int. 42 (2016) 9375-9381.

[16] S. Gartiser, L. Brinker, T. Erbe, K. Kümmerer, R. Willmund, Belastung von Krankenhausabwasser mit gefährlichen Stoffen im Sinne § 7a WHG, Acta Hydroch. Hydrob. 24 (1996) 90-97.

[17] C. Boillot, C. Bazin, F. Tissot-Guerraz, J. Droguet, M. Perraud, J.C. Cetre, D. Trepo, Y. Perrodin, Daily Physicochemical, Microbiological and Ecotoxicological Fluctuations of a Hospital Effluent According to Technical and Care Activities, Sci. Total Environ. 403 (2008) 113-129.

[18] A. Dalhoff, S. Schubert, A. Vente, Pharmacodynamics of Finafloxacin, Ciprofloxacin, and Levofloxacin in Serum and Urine against TEM- and SHV-Type ExtendedSpectrum- $\beta$-Lactamase-Producing Enterobacteriaceae Isolates from Patients with Urinary Tract Infections, Antimicrob. Agents Chemother. 61 (2017) e02446-16.

[19] M. Kosmulski, pH-Dependent Surface Charging and Points of Zero Charge II. Update, J. Colloid Interface Sci. 275 (2004) 214-224.

[20] M. Kosmulski, pH-Dependent Surface Charging and Points of Zero Charge, J. Colloid Interface Sci. 298 (2006) 730-741.

[21] M. Müllner, A.H.E. Müller, Cylindrical Polymer Brushes - Anisotropic Building Blocks, Unimolecular Templates and Particulate Nanocarriers, Polymer 98 (2016) 389-401.

[22] J. Yuan, Y. Xu, A. Walther, S. Bolisetty, M. Schumacher, H. Schmalz, M. Ballauff, A.H.E. Müller, Water-Soluble Organo-Silica Hybrid Nanowires, Nat. Mater. 7 (2008) 718-722.

[23] Y. Xu, J. Yuan, B. Fang, M. Drechsler, M. Müllner, S. Bolisetty, M. Ballauff, A.H.E. Müller, Hybrids of Magnetic Nanoparticles with Double-Hydrophilic Core/Shell Cylindrical Polymer Brushes and Their Alignment in a Magnetic Field, Adv. Funct. Mater. 20 (2010) 4182-4189.

[24] S.S. Sheiko, B.S. Sumerlin, K. Matyjaszewski, Cylindrical Molecular Brushes: Synthesis, Characterization and Properties, Prog. Polym. Sci. 33 (2008) 759-785.

[25] M. Müllner, T. Lunkenbein, N. Miyajima, J. Breu, A.H.E. Müller, A Facile Polymer Templating Route Toward High-Aspect-Ratio Crystalline Titania Nanostructures, Small 8 (2012) 2636-2640. 
[26] M. Müllner, T. Lunkenbein, M. Schieder, A.H. Gröschel, N. Miyajima, M. Förtsch, J. Breu, F. Caruso, A.H.E. Müller, Template-Directed Mild Synthesis of Anatase Hybrid Nanotubes within Cylindrical Core-Shell-Corona Polymer Brushes, Macromolecules 45 (2012) 6981-6988.

[27] M. Schieder, T. Lunkenbein, T. Martin, W. Milius, G. Auffermann, J. Breu, Hierarchically Porous Tungsten Oxide Nanotubes with Crystalline Walls Made of the Metastable Orthorhombic Polymorph, J. Mater. Chem. A 1 (2013) 381-387.

[28] T. Lunkenbein, D. Rosenthal, T. Otremba, F. Girgsdies, Z. Li, H. Sai, C. Bojer, G. Auffermann, U. Wiesner, J. Breu, Access to Ordered Porous Molybdenum Oxycarbide/Carbon Nanocomposites, Angew. Chem. Int. Ed. 51 (2012) 12892-12896.

[29] M. Schieder, C. Bojer, J. vom Stein, S. Koch, T. Martin, H. Schmalz, J. Breu, T. Lunkenbein, Template Removal via Boudouard Equilibrium Allows for Synthesis of Mesostructured Molybdenum Compounds, Angew. Chem. Int. Ed. (2017), DOI: 10.1002/anie.201610786.

[30] C. Bojer, J. Schöbel, T. Martin, M. Ertl, H. Schmalz, J. Breu, Clinical Wastewater Treatment: Photochemical Removal of an Anionic Antibiotic (Ciprofloxacin) by Mesostructured High Aspect Ratio ZnO Nanotubes, Appl. Catal. B Environ. 204 (2017) 561-565.

[31] L. Sun, D. Zhao, Z. Song, C. Shan, Z. Zhang, B. Li, D. Shen, Gold Nanoparticles Modified ZnO Nanorods with Improved Photocatalytic Activity, J. Colloid Interface Sci. 363 (2011) 175-181. 